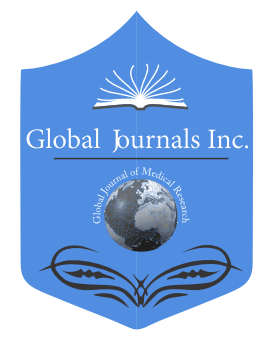

\title{
Results of Comparison of Two Types of Olfactory Recognition Tests Performed on 112 Peoples. -34 High School Students, 55 University Students, and 23 Middle-Aged
}

By Naomi Katayama, Syoko Kondo, Yui Ando, Youko Ashihara, Nene Kawano, Mrika Shibuya, Misaki Nanao, Inori Mase, Minami Abe, Marina Kouno \& Yuuna Narimoto

Nagoya Women's University

Abstract- The olfactory cognitive test is not commonly used. Still, it required in the future because it reported that and olfactory disorder appears as an initial symptom of Alzheimer's dementia or COVID-19 infection. There are several types of odor inspection kits used for testing, but in Japan, there are odor sticks, open essences, T\&T olfactometry, etc. This time, we report that we conducted an olfactory cognitive test using Odor Sticks and Open Essence on healthy 112 peoples (34 high school students, 55 university students, and 23 middle-aged). The Open Essence (made by FUJIFILM) has the smell as same as the Odor Stick Identification Test (OSIT-J). The odor Stick (made by Daiichi Pharmaceutical industry Co., Ltd.) and the open essence include the aromas as curry, perfume, Japanese cypress, India ink, menthol, rose, wood, stynkysocks/sweat, roasted garlic, condensed milk, gas for cooking, and Japanese mandarin aromas. This 12 different odorants perception is not necessarily culture-free; the Japanese version employed.

Keywords: olfaction test, odor stick, open essence, cognition, age.

GJMR-K Classification: NLMC Code: WV 140

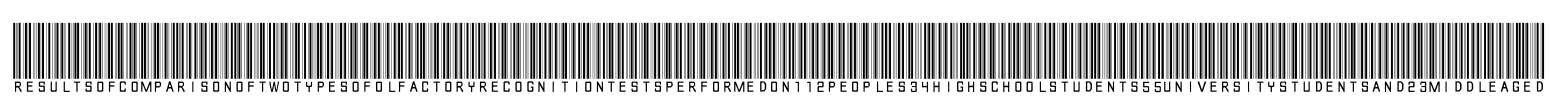

Strictly as per the compliance and regulations of:

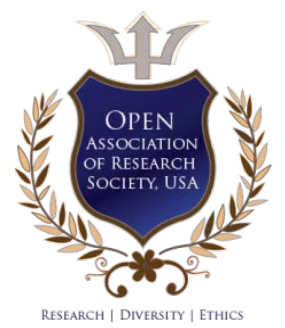

(c) 2020. Naomi Katayama, Syoko Kondo, Yui Ando, Youko Ashihara, Nene Kawano, Mrika Shibuya, Misaki Nanao, Inori Mase, Minami Abe, Marina Kouno \& Yuuna Narimoto. This is a research/review paper, distributed under the terms of the Creative Commons Attribution-Noncommercial 3.0 Unported License http://creativecommons.org/licenses/by-nc/3.0/), permitting all noncommercial use, distribution, and reproduction in any medium, provided the original work is properly cited. 


\title{
Results of Comparison of Two Types of Olfactory Recognition Tests Performed on 112 Peoples. -34 High School Students, 55 University Students, and 23 Middle-Aged
}

\author{
Naomi Katayama ${ }^{\alpha}$, Syoko Kondo ${ }^{\circ}$, Yui Ando $^{\rho}$, Youko Ashihara $^{\omega}$, Nene Kawano ${ }^{\ddagger}$, Mrika Shibuya $^{\S}$, \\ Misaki Nanao ${ }^{x}$, Inori Mase ${ }^{\vee}$, Minami Abe $^{\ominus}$, Marina Kouno ${ }^{`} \&$ Yuuna Narimoto ${ }^{f}$
}

Abstract- The olfactory cognitive test is not commonly used. Still, it required in the future because it reported that and olfactory disorder appears as an initial symptom of Alzheimer's dementia or COVID-19 infection. There are several types of odor inspection kits used for testing, but in Japan, there are odor sticks, open essences, T\&T olfactometry, etc. This time, we report that we conducted an olfactory cognitive test using Odor Sticks and Open Essence on healthy 112 peoples (34 high school students, 55 university students, and 23 middleaged). The Open Essence (made by FUJIFILM) has the smell as same as the Odor Stick Identification Test (OSIT-J). The odor Stick (made by Daiichi Pharmaceutical industry Co., Ltd.) and the open essence include the aromas as curry, perfume, Japanese cypress, India ink, menthol, rose, wood, stynkysocks/sweat, roasted garlic, condensed milk, gas for cooking, and Japanese mandarin aromas. This 12 different odorants perception is not necessarily culture-free; the Japanese version employed. The average \pm standard deviation of the number of olfactory recognition using Open Essence was $8.4 \pm 2.0$ for high school students, $8.4 \pm 1.5$ for university students, and $7.8 \pm 2.2$ for middle-age. The average \pm standard deviation of the number of olfactory recognition using Odor Stick was $8.8 \pm 1.7$ for high school students, $9.9 \pm 1.5$ for university students, and $9.1 \pm 1.9$ for middle-age. There were no significant differences between the two olfactory cognitive tests in high school students. However, the university students and the middle-age had a statistically significant difference in the cognitive score between Open Essence and Odor Stick. The Odor Stick score is better than the Open Essence. When odors examined individually, all data showed that menthol and stinky socks/ sweaty had a recognition rate of $80 \%$ or higher. And odors were examined individually; all data showed that India Ink had are cognition rate of less than $70 \%$. The odors that differed in the number of cognition in the two olfactory cognition tests were the Stir-fried garlic, and the Odor Stick was better than the Open Essence. Keywords: olfaction test, odor stick, open essence, cognition, age.

Author $\alpha \rho \omega ¥ \S \chi v \theta \zeta £$ : Nagoya Women's University, Nagoya City, Japan.e-mail:naomik@nagoya-wu.ac.jp

Author a: Graduate School of Nagoya Women's University, Nagoya City, Japan.

Author $\sigma:$ Watanabe Hospital, Mihama town, Noma, Aichi, Japan.

\section{INTRODUCTION}

$\longrightarrow$ ecent years, many researchers have reported odor research, but few reports by age in healthy people. Also, since odors are closely related to daily life, there are differences in odors that are often contacted from birth to death in each country. Therefore, each country had its odor inspection kit (smell related to the life of the country). This time, I decided to conduct the Japanese odor test using the Japanese odor kit (Odor Stick: Daiichi Pharmaceutical industry Co., Ltd., and Open Essence: FUJIFILM). The Odor Stick Identification Test (OSIT-J) was used to assess odor perception for many years for our study. This test possesses high reliability and validity 1). The procedure resembles that of the San Diego Odor Identification Test 2). The aromas used in the OSIT-J includes curry, perfume; Japanese cypress, India ink, menthol, rose, wood, stinky socks/sweat, roasted garlic, condensed milk, gas for cooking, and Japanese mandarin aromas 2). This 12 different odorants perception is not necessarily culture-free, the Japanese version was employed 3, 4). Each fragrance enclosed in microcapsules made of melamine resin3,4). Therefore, in this study, I recruited high school students in their teens, university students in their 20s, and middle-age in their 30 s to 40 s, and compared the data by conducting two kinds of olfactory cognitive tests.

\section{il. Materials and Methods}

\section{a) Participants}

The participants were 34 high school students and 55 university students and 24 middle-age $(n=112)$ who voluntarily participated in olfactory tests (Table 1 ). The average age of high school students was $17.03 \pm 10.67$, university students were $20.46 \pm 0.54$, and middle-ages were $47.14 \pm 2.61$. 
Table 1: Basic information of participants (Average of age, height, and weight)

\begin{tabular}{lccc}
\hline \hline & Age & Height & Weight \\
\cline { 2 - 4 } & Average \pm S D & Average \pm S D & Average \pm S D \\
\hline High school students $(n=34)$ & $17.03 \pm 0.67$ & $157.17 \pm 5.12$ & $48.27 \pm 6.60$ \\
University students $(n=55)$ & $20.46 \pm 0.54$ & $157.76 \pm 6.20$ & $50.35 \pm 4.48$ \\
Middle-age $(n=23)$ & $47.14 \pm 2.61$ & $159.71 \pm 8.43$ & $54.94 \pm 10.10$ \\
\hline \hline
\end{tabular}

\section{$\mathrm{SD}=$ Standard Deviation}

\section{b) Assessment of odor identification}

In this test, two kinds of olfactory recognition tests performed on the same participant. The test kit used is the Odor Stick (Daiichi Pharmaceutical industry Co., Ltd.,) and Open Essence (FUJIFILM). There two types of olfactory cognitive test kits consist of 12 types of odors. This test possesses high reliability and validity 1). The basic procedure resembles that of the San Diego Odor Identification Test 2).Both kit includes curry, perfume, Japanese cypress, India ink, menthol, rose, wood, stinky socks/sweat, roasted garlic, condensed milk, gas for cooking, and Japanese mandarin aromas. This 12 different odorants perception is not necessarily culture-free; the Japanese version was employed 3,4). Each fragrance enclosed in microcapsules made of melamine resin3,4).Each correct answer was scored as one point with the total performance score ranging from 0 to 12 points5,6). We defined it as follows: normal range as more than 6 points, borderline as 3 to 5 points, and abnormal as less than 2 points5,6). All of these methods are the same as in the previously reported paper 5,6).

\section{c) Statistical processing}

The test results were confirmed to be normal distribution by F-test. Data that distributed compared with Student-t without correlation of parametric test. The data that was not distributed compared without correlated Mann-Whitney test of the non-parametric test. In comparing the taste test and the olfactory test result performed on the same participant, with correlated Wilcoxon test of the non-parametric test.

\section{d) Ethical review board}

This study conducted with the approval of the Ethical Review Board (Nagoya women's university 'hitowomochiitakennkyuunikansuruiinnkai').

The approval number is $30-11$.

\section{Results}

a) Odor identification (number of the correct answers)

Tables 2, 3, and 4 show the results of two types of olfactory cognitive tests for high school students, university students, and middle-age. Tables 5 and 6 show the average and standard deviation of the number of olfactory cognition in each group for each olfactory cognitive test. No one had less than two olfactory cognition sat any age. There is no difference in the number of olfactory cognition among the groups in each olfactory cognitive test. However, there are differences when looking at the two types of test results in university students and middle-aged people.

Table 2: Comparison of recognition numbers of two of olfactory test in high school students (Number of people) $(n=34)$

\begin{tabular}{|c|c|c|c|c|c|c|c|c|c|c|c|c|c|}
\hline & 0 & 1 & 2 & 3 & 4 & 5 & 6 & 7 & 8 & 9 & 10 & 11 & 12 \\
\hline Open essence & 0 & 0 & 0 & 0 & 1 & 2 & 4 & 2 & 10 & 6 & 5 & 1 & 3 \\
\hline Odor stick & 0 & 0 & 0 & 0 & 1 & 2 & 0 & 2 & 6 & 11 & 7 & 5 & 0 \\
\hline
\end{tabular}

Table 3: Comparison of recognition numbers of two of olfactory test in university students (Number of people) $(n=55)$

\begin{tabular}{llllllllllllll}
\hline & $\mathbf{0}$ & $\mathbf{1}$ & $\mathbf{2}$ & 3 & 4 & 5 & 6 & 7 & $\mathbf{8}$ & $\mathbf{9}$ & 10 & 11 & 12 \\
\hline Open essence & $\mathbf{0}$ & $\mathbf{0}$ & $\mathbf{0}$ & $\mathbf{1}$ & $\mathbf{1}$ & $\mathbf{0}$ & 2 & 7 & 17 & 15 & 10 & 2 & $\mathbf{0}$ \\
Odor stick & $\mathbf{0}$ & $\mathbf{0}$ & $\mathbf{0}$ & $\mathbf{0}$ & $\mathbf{0}$ & $\mathbf{0}$ & 1 & 4 & 4 & $\mathbf{8}$ & 15 & 17 & 6 \\
\hline
\end{tabular}

Table 4: Comparison of recognition numbers of two of olfactory test in middle age (Number of people) $(n=23)$

\begin{tabular}{lccccccccccccc}
\hline & 0 & 1 & 2 & 3 & 4 & 5 & 6 & 7 & 8 & 9 & 10 & 11 & 12 \\
\hline Open essence & 0 & 0 & 0 & 1 & 1 & 1 & 4 & 4 & 2 & 4 & 5 & 1 & 0 \\
Odor stick & 0 & 0 & 0 & 0 & 1 & 0 & 0 & 5 & 2 & 3 & 6 & 6 & 0 \\
\hline \hline
\end{tabular}


Table 5: Results of olfactory cognition test using open essence

(Average number of recognition \pm Standard Deviation)

\begin{tabular}{lc}
\hline \hline & Numver of recognition \pm Standard Deviation \\
\hline High school students $(n=34)$ & $8.4 \pm 2.0$ \\
University students $(n=55)$ & $8.4 \pm 1.5$ \\
Middle-age $(n=23)$ & $7.8 \pm 2.2$ \\
\hline \hline
\end{tabular}

Table 6: Results of olfactory cognition test using odor stick

(Average number of recognition \pm Standard Deviation)

\begin{tabular}{lc}
\hline \hline & Numver of recognition \pm Standard Deviation \\
\hline High school students $(\mathrm{n}=34)$ & $\mathbf{8 . 8} \pm 1.7$ \\
University students $(\mathrm{n}=55)$ & $9.9 \pm 1.5$ \\
Middle-age $(\mathrm{n}=23)$ & $9.1 \pm 1.9$ \\
\hline \hline
\end{tabular}

b) Statistical comparison

Tables7, 8 and 9 show a comparison of the olfactory cognitive test results using statistical methods. There were no stutisttically significant differences between the two olfactory cognitive test results in high school students. However, there was a statistically significant difference between the two types of olfactory cognitive test results between university students and middle-aged, and the odor stick had a higher degree of recognition than Open Essence. Furthermore, when comparing between groups, the Open Essence olfactory cognition test showed n statistically significant difference between all groups. However, there was a statistically significant difference between university students and other groups in the Odor Stick.

Table 7: Statistical comparison of olfactory recognition number of the open essence test and odor stick test (High school students, University students and Middle-age)

\begin{tabular}{|c|c|c|c|c|c|c|}
\hline \multirow[t]{2}{*}{ Odor idetification } & \multicolumn{2}{|c|}{ High school students (n=34) } & \multicolumn{2}{|c|}{ University students $(n=55)$} & \multicolumn{2}{|c|}{ Middle-age $(n=23)$} \\
\hline & Open essence & Odor stick & Open essence & Odor stick & Open essence & Odor stick \\
\hline Average number of recognition \pm Standard Deviation & $8.4 \pm 2.0$ & $8.8 \pm 1.7$ & $8.4 \pm 1.5$ & $9.9 \pm 1.5$ & $7.8 \pm 2.2$ & $9.1 \pm 1.9$ \\
\hline F test & \multicolumn{2}{|c|}{$P=0.154$} & \multicolumn{2}{|c|}{$P=0.443$} & \multicolumn{2}{|c|}{$P=0.261$} \\
\hline Paired Student-t test & \multicolumn{2}{|c|}{$P=0.309$} & \multicolumn{2}{|c|}{$\mathrm{P}=0.0001^{* *}$} & \multicolumn{2}{|c|}{$\mathrm{P}=0.005^{* *}$} \\
\hline
\end{tabular}

Table 8: Statistical comparison of olfactory recognition number of the open essence test

(High school students vs University students, University students vs Middle-age, Middle-age vs High sch. students)

\begin{tabular}{|c|c|c|c|c|c|c|}
\hline \multirow[t]{2}{*}{ Odor idetification } & \multicolumn{2}{|c|}{ Open essence } & \multicolumn{2}{|c|}{ Open essence } & \multicolumn{2}{|c|}{ Open essence } \\
\hline & Hight school students & University students & University students & Middle-age & Middle-age & Hight school students \\
\hline Average number of recognition \pm Standard Deviation & $8.4 \pm 2.0$ & $8.4 \pm 1.5$ & $8.4 \pm 1.5$ & $7.8 \pm 2.2$ & $7.8 \pm 2.2$ & $8.4 \pm 2.0$ \\
\hline F test & \multicolumn{2}{|c|}{$P=1.00$} & \multicolumn{2}{|c|}{$\mathrm{P}=0.031$ * } & \multicolumn{2}{|r|}{$P=0.396$} \\
\hline Paired Student-t test & \multicolumn{2}{|c|}{$P=0.999$} & & & \multicolumn{2}{|r|}{$\mathbf{P}=\mathbf{0 . 1 7 0}$} \\
\hline Mann-Whitny test & \multicolumn{6}{|c|}{$P=0.129$} \\
\hline
\end{tabular}

Table 9: Statistical comparison of olfactory recognition number of the open odor stick test (High school students vs University students, University students vs Middle-age, Middle-age vs High sch. students)

\begin{tabular}{|c|c|c|c|c|c|c|}
\hline \multirow[t]{2}{*}{ Odor idetification } & \multicolumn{2}{|c|}{ Odor stick } & \multicolumn{2}{|c|}{ Odor stick } & \multicolumn{2}{|r|}{ Odor stick } \\
\hline & Hight school students & University students & University students & Middle-age & Middle-age & Hight school students \\
\hline Average number of recognition \pm Standard Deviation & $8.8 \pm 1.7$ & $9.9 \pm 1.5$ & $9.9 \pm 1.5$ & $9.1 \pm 1.9$ & $9.1 \pm 1.9$ & $9.1 \pm 1.9$ \\
\hline$F$ test & \multirow{2}{*}{\multicolumn{2}{|c|}{$P=0.154$}} & \multicolumn{2}{|c|}{$P=0.103$} & \multicolumn{2}{|r|}{$P=0.316$} \\
\hline Paired Student-t test & & & \multicolumn{2}{|c|}{$\mathrm{P}=0.026^{*}$} & \multicolumn{2}{|r|}{$P=0.649$} \\
\hline
\end{tabular}

c) Odor identification (percentage of each smell)

Next, Tables 10, 11, and 12 show the results of individually examining each of the 12 odors. Curry was the smell that high school students, university students, and middle-age showed the highest olfactory perception.

When odors examined individually, all data showed that menthol and stinky socks/ sweaty had a recognition rate of $80 \%$ or higher. And odors examined individually; all data showed that India Ink had are rcognition rate of less than $70 \%$. The odors that differed in the number of cognition in the two olfactory cognition tests were the Stir-fried garlic, and the odor stick was better than the open essence. 
Table 10: Comparison of results of different odors of two cognitive test in high school students (\%) $(\mathrm{n}=34)$

\begin{tabular}{|c|c|c|c|c|c|c|c|c|c|c|c|c|}
\hline & India Ink & Wood & Perfume & Menthol & Mandarin orange & Curry & Household gas & Rose & Cypress & Stinky socks/Sweaty & Condensed milk & Stir-fried garlic \\
\hline Open essence & 52.9 & 61.8 & 58.8 & 97.1 & 44.1 & 97.1 & 70.6 & 85.3 & 91.2 & 91.2 & 73.5 & 23.5 \\
\hline Odor stick & 44.1 & 50 & 23.5 & 85.3 & 97.1 & 97.1 & 58.8 & 88.2 & 76.5 & 94.1 & 70,6 & 97.1 \\
\hline
\end{tabular}

Table 11: Comparison of results of different odors of two cognitive test in university students $(\%)(n=55)$

\begin{tabular}{lcccccccccccc}
\hline & India Ink & Wood & Perfume & Menthol & Mandarin orange & Curry & Household gas & Rose & Cypress & Stinky socks/Sweaty & Condensed milk & Stir-fried garlic \\
\hline Open essence & 45.5 & 74.5 & 58.2 & 94.5 & 21.8 & 96.4 & 81.8 & 83.6 & 83.6 & 96.4 & 83.6 & 20.0 \\
Odor stick & 61.8 & 63.6 & 67.3 & 90.9 & 87.3 & 89.1 & 87.3 & 92.7 & 78.2 & 96.4 & 89.1 & 94.5 \\
\hline
\end{tabular}

Table 12: Comparison of results of different odors of two cognitive test in middle age students $(\%)(n=23)$

\begin{tabular}{|c|c|c|c|c|c|c|c|c|c|c|c|c|}
\hline & India Ink & Wood & Perfume & Menthol & Mandarin orange & Curry & Household gas & Rose & Cypress & "Stinky socks/Sweaty & Condensed milk & Stir-fried garlic \\
\hline Open essence & 47.8 & 56.5 & 82.6 & 87 & 95.7 & 73.9 & 73.9 & 73.9 & 73.9 & 82.6 & 56.5 & 26.1 \\
\hline Odor stick & 65.2 & 69.6 & 17.4 & 95.7 & 91.3 & 100 & 87 & 91.3 & 73.9 & 100 & 60.9 & 100 \\
\hline
\end{tabular}

\section{Discussion}

The result of the elderly reported by Katayama et al. In the past showed that the olfactory cognitive test results gradually decreased in both males and females after the '50s. This time, I reported the results of olfactory cognition tests in the teens, 20 s, and 30 s to 40s. The results of olfactory cognition tests were almost the same in the younger age group, and most of them recognized eightor more of 12 odors. The results of the olfactory cognition test using open essence showed no statistically significant difference in the cognitive results among high school students, university students, and middle-age. However, there was a statistically significant difference between the results of university students and middle-age in the odor stick compared to high school students. When odors examined individually, all data showed that menthol and stinky socks/ sweaty had a recognition rate of $80 \%$ or higher. And odors were examined individually; all data showed that India Ink had are cognition rate of less than $70 \%$. The odors that differed in the number of cognition in the two olfactory cognition tests were the Stir-fried garlic, and the odor stick was better than the open essence. Also, since the results of the olfactory cognition test in the younger generation are a small number, I would like to continue the test and collect the data for re-examination.

\section{Conclusions}

This time, we report that we conducted an olfactory cognitive test using odor sticks and open essence on healthy 112 peoples (34 high school students, 55 university students, and 23 middle-aged). The Open Essence (made by FUJIFILM) has the smell as same as the odor Stick Identification Test (OSIT-J).

The average \pm standard deviation of the number of olfactory recognition using open essence was $8.4 \pm 2.0$ for high school students, $8.4 \pm 1.5$ for university students, and $7.8 \pm 2.2$ for middle-age. The result of the odor stick was $8.8 \pm 1.7$ for high school students, $9.9 \pm 1.5$ for university students, and $9.1 \pm 1.9$ for middle-age. There were no significant differences between the two olfactory cognitive tests in high school students. However, the university students and the middle-age had a statistically significant difference in the cognitive score between open essence and Odor Stick. The Odor Stick score is better than the Open Essence. When odors examined individually; all data showed that menthol and stinky socks/ sweaty had arecognition rate of $80 \%$ or higher. And odors examined individually; all data showed that India Ink had are cognition rate of less than $70 \%$. The odors that differed in the number of cognition in the two olfactory cognition tests were the Stir-fried garlic, and the odor stick was better than the open essence. In the future, I would like to increase the number of participants and report the results of participants and report the results of olfactory cognition tests by age and sex.

\section{ACKNOWLeDgements}

This study was supported by the research aid of Choju-iryo-kenkyu-kaihatsuhi 30-14 and the Japanese Society of Taste Technology, 2019.

\section{References Références Referencias}

1. Kobayashi M (2005). The odor Stick Identification Test for the Japanese (OSIT-J): Clinical suitability for patients suffering from olfactory disturbance. Chemical Senses, 30(Suppl 1): i216-i217.

2. Murphy C, Anderson AJ, Markinson S (1994). Psychophysical assessment of chemosensory disorders in clinical populations. In K. Kurihara, N. Suzuki, \& H. Ogawa (Eds.) Olfaction and Taste. Tokyo: Springer Verlag Tokyo. pp. 609-613

3. Kobayashi M, Reiter ER, DiNardo JL, Costanzo MR (2007). A new clinical olfactory function test: cultural influence. Arch. Otolaryngol-Head Neck Surg. 133(4): 331-336.

4. Kobayashi M, Saito S, Kobayakawa T, Deguchi Y, Costanzo RM (2006). Cross-cultural comparison of data using the Odor Stick Identification Test for Japanese (OSIT-J). Chem. Senses. 31(4): 335-342.

5. Katayama N, Kondo S, Ootake H et al (2018). Odour and Salt Taste Identification in Older Adults: 
Evidence from the Yakumo Study in August, 2018. Acade. J. Med. Plants 7(3) 066-071.

6. Naomi Katayama, Shoko Kondo, Satofumi Sugimoto, Tadao Yoshida, Masaaki Teranishi, Michihiko Sone3 Yasushi Fujimoto, Hironao Otake, Hirokazu Suzuki, Takafumi Nakada, Naoki Saji, Seiichi Nakata and Tsutomu Nakashima (2019. Odour and salt taste identification in older adults: Evidence from the Yakumo. Academia Journal of Medicinal Plant, 8(3): 030-035. 